\title{
Efecto del riego deficitario controlado en las tasas de crecimiento del fruto de pera (Pyrus communis L.), var. Triunfo de Viena
}

\section{Effect of controlled deficit irrigation on the fruit growth rates of the Triunfo de Viena pear (Pyrus communis L.) variety}

MARÍA JAQUELINE MOLINA-OCHOA

JAVIER ENRIQUE VÉLEZ-SÁNCHEZ1, 3

PEDRO RODRÍGUEZ²

Frutos de pera en fructificación en Sesquilé (Cundinamarca).

Foto: J.E. Vélez-Sánchez

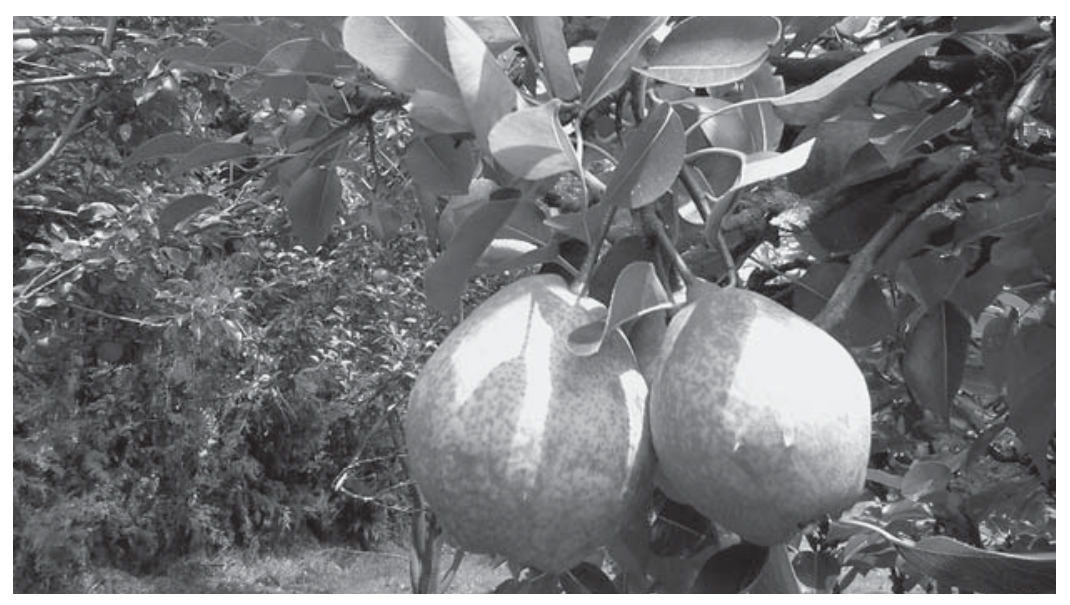

\section{RESUMEN}

De octubre de 2011 a abril de 2012 se realizó un experimento de riego deficitario controlado (RDC) en un cultivo de peral, variedad Triunfo de Viena que consistió en la aplicación de tres tratamientos de riego: el primer tratamiento, el control, regado durante todo el año al 100\% de la evapotranspiración potencial (ETc), el segundo (T1) y tercer tratamiento (T2), se regaron durante todo el año al 100\% de la (ETc) y durante la etapa de crecimiento rápido del fruto al $67 \%$ y $55 \%$ de la (ETc), respectivamente. La (ETo) se determinó mediante la ecuación de Penman-Monteith. Los resultados indican que ninguno de los tratamientos difirió significativamente del control en cuanto al crecimiento del fruto, obteniéndose un ahorro de agua del 33 y $45 \%$, respecto al control en el T1 y T2, respectivamente durante la aplicación diferencial del riego, siguiendo una curva sigmoide ajustada a una ecuación logística de tres parámetros.

Palabras clave adicionales: evapotranspiración potencial, ahorro de agua, curva sigmoide.

Facultad de Ingeniería, Departamento de Ingeniería Civil e Ingeniería Agrícola, Universidad Nacional de Colombia, Bogotá (Colombia).

2 Departamento de Fisiología y Bioquímica Vegetal, Instituto Nacional de Ciencias Agrícolas (INCA), Universidad Agraria de la Habana, San José de Las Lajas, Mayabeque (Cuba).

3 Autor para correspondencia. jevelezs@unal.edu.co 


\section{ABSTRACT}

From October, 2011 to April, 2012, an experiment was carried out with deficit irrigation (RDC) in a crop of the Triunfo de Vienna pear variety, which consisted of three irrigation treatments: control, irrigated throughout the year at $100 \%$ of the potential evapotranspiration (ETc); treatments (T1) and (T2) were irrigated throughout the year at 100\% (ETc) except during the period of rapid fruit growth when $67 \%$ and $55 \%$ of the (ETc) were used, respectively. The evapotranspiration (ETo) was determined with the PenmanMonteith equation. The results indicated that none of the treatments differed significantly from the control in terms of fruit growth, yielding a water savings of 33 to $45 \%$ as compared to the control for T1 and T2, respectively, with the differential irrigation application, following a sigmoid adjusted to a three-parameter logistic equation curve.

Additional key words: potential evapotranspiration, water savings, sigmoid curve.

Fecha de recepción: 27-02-2015

Aprobado para publicación: 20-11-2015
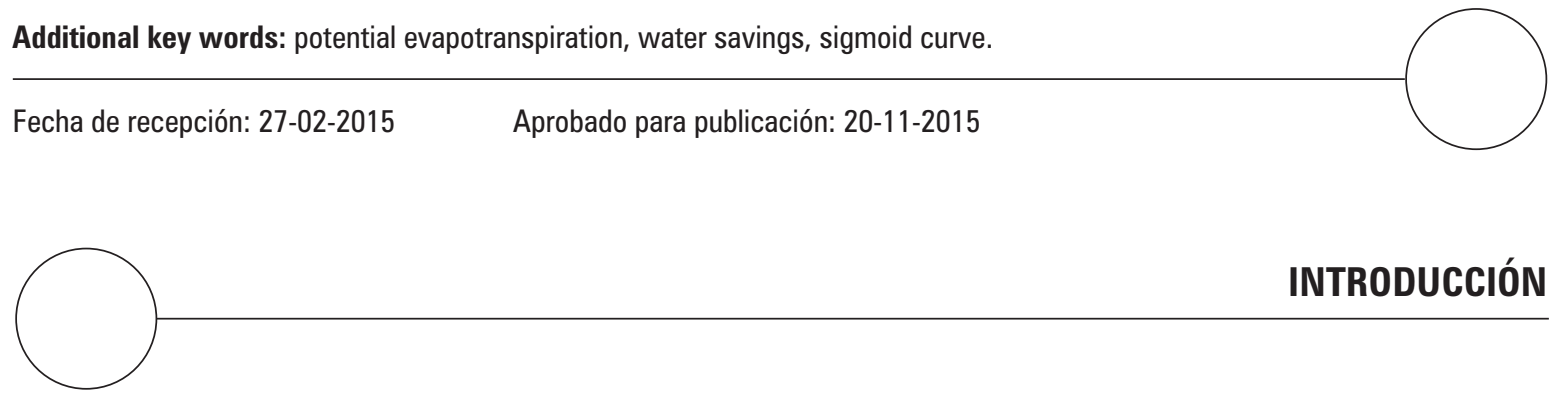

INTRODUCCIÓN

El riego en frutales es importante para suplir las necesidades hídricas de las plantas cuando la precipitación no es suficiente. Debido a las ventajas del consumo de frutas frescas, el área a nivel mundial se ha incrementado y específicamente en peral (Pyrus communis L.), por sus propiedades medicinales (Li et al., 2014).

El riego deficitario controlado (RDC) consiste en la "reducción de aportes de agua en aquellas fases del cultivo donde es menos sensible al estrés hídrico" (Chalmers et al., 1986). Varios investigadores han estudiado esta técnica en cultivos de pera en la primera etapa de desarrollo del fruto (Chalmers et al., 1986; Marsal et al., 2002; Wu et al., 2013) y después de la cosecha (Naor et al., 2006; Marsal et al., 2012), con el propósito de obtener ahorros significativos de agua sin afectar la producción y calidad del fruto.

El riego deficitario es útil en los casos en los que la demanda hídrica no puede ser satisfecha con los recursos disponibles (Girona, 1996). Behboudian et al., 1994; Alegre et al., 2002; Marsal et al., 2002; Intrigliolo et al., 2006; Ningbo et al., 2009; Vélez et al., 2011 han trabajado sobre este tema en olivo, pera, ciruela, cítricos y otros frutales, para determinar condiciones favorables o desfavorables de su aplicación, estableciendo rangos en los que se obtienen mejores resultados. En jujube (Zizyphus jujuba), aplicando en el periodo de apertura de brotes, un déficit moderado, redujo el agua de riego y restringió el crecimiento vegetativo, aumentando ligeramente o manteniendo el rendimiento de fruta (Ningbo et al., 2009).

Las curvas de crecimiento en los vegetales refleja el comportamiento de una planta en un ecosistema particular con respecto al tiempo. Las tasas de crecimiento comúnmente utilizadas para el análisis, son la tasa absoluta (TAC) que indica cambio de tamaño por unidad de tiempo y la relativa (TRC) que indica la variación de tamaño por unidad de tamaño inicial (Hunt, 1990). A pesar de que la duración e intensidad de las fases de crecimiento y desarrollo del fruto dependen ampliamente del genotipo, existe una estrecha relación con: la temperatura ambiente, humedad relativa y estado hídrico de la planta (Casierra-Posada, 2012).

El fruto de pera crece, igualmente como la manzana, de forma sigmoidal simple (Jackson, 2003). 
En frutales de pepita, Casierra et al. (2003) definieron tres fases para el crecimiento y desarrollo de frutos: (1) periodo de multiplicación celular, que dura de cuatro a cinco semanas; (2) crecimiento acelerado por elongación celular, que puede durar de cinco a diez semanas, y (3) maduración, la cual comienza realmente antes de la culminación del periodo anterior, estas mismas fases son las expuestas por Fischer et al. (2012), para la curva de crecimiento sigmoide simple.

Se considera que el crecimiento y la productividad de los cultivos es proporcional al uso eficiente del agua y un déficit hídrico severo reduce normalmente la producción final, por el contrario, en algunos casos un estrés moderado puede llegar a mejorar la calidad (Vélez et al., 2007a).

El estrés ocasionado en el periodo de crecimiento o expansión celular de la pera reduce el potencial de alcanzar el tamaño comercial de frutas en la cosecha (Marsal et al., 2010). Sin embargo, Naor et al. (2006) encontraron que la reducción del riego de forma moderada después de la cosecha en pera "Spadona" aumentó el rendimiento en la temporada siguiente, teniendo en cuenta que la respuesta se determina por el nivel del estrés aplicado, ya que cuando el estrés fue severo el rendimiento se redujo.

Un déficit hídrico en árboles adultos del peral, durante la etapa de división celular, que corresponde al periodo de crecimiento vegetativo, puede restringir el crecimiento de los tallos sin mostrar diferencia en el tamaño de la fruta (Wu et al., 2013).

En frutales caducifolios en condiciones de clima mediterráneo la falta de agua constituye un factor limitante del crecimiento del fruto (Goldschmidt y Monselise, 1977) y en general de la producción (Levy et al., 1979). Un periodo crítico es la fase inicial de crecimiento del fruto, donde aumenta la caída y provoca la disminución de la tasa de crecimiento, determinando el tamaño final del mismo (Shalhevet et al., 1979).
Las mediciones del diámetro del fruto como indicador del momento en que debe aplicarse el riego, fueron recomendadas ya desde tiempos antiguos. Aplicando el riego cuando el crecimiento del fruto disminuye o se detiene, se consigue que el tamaño del árbol y la tasa de crecimiento del fruto sea menor, de tal forma que en la etapa de crecimiento rápido, luego de aplicar el riego, los frutos se recuperan sin afectar la producción, que resulta igual a la de árboles bien regados durante todo el crecimiento. Ebel et al. (1995) recomendaron terminar el RDC en manzano antes de que el crecimiento del fruto fuera inferior al de la curva estándar de crecimiento obtenida en condiciones sin restricción de agua, para de esta forma disminuir el crecimiento vegetativo sin que se afectara la producción ni el tamaño de los frutos. El inconveniente para utilizar el crecimiento del fruto como indicador en la programación del riego, es la alta variabilidad entre frutos y el que no haya siempre frutos, tampoco permite utilizar este parámetro para programar el riego.

La medida indirecta del contenido hídrico de la planta se ha realizado mediante diversas técnicas. Por medio de microvariaciones del espesor de las hojas y de los diámetros de ramas, tallos y frutos (Kozlowski y Winguet, 1964; Kozlowski, 1967; Klepper et al., 1971; Huguet, 1985; Intrigliolo y Castel, 2006; Vélez et al., 2007b).

El objetivo del presente estudio fue determinar el efecto de la disminución del volumen de agua aplicado durante la etapa de crecimiento rápido del fruto en el crecimiento vegetativo y del fruto de la pera variedad Triunfo de Viena.

\section{MATERIALES Y MÉTODOS}

El experimento se realizó desde octubre de 2011 hasta abril de 2012, en el municipio de Sesquilé (Cundinamarca, Colombia), a 2.595 msnm, con temperatura media de $14^{\circ} \mathrm{C}$. La parcela experimental tiene una superficie aproximada de 0,32 
ha, plantada en 1998 con peral var. Triunfo de Viena, con una densidad de siembra de $4 \times 4 \mathrm{~m}$.

El suelo típico de la zona es de textura franco arcillosa. La capacidad de campo y el punto de marchitez permanente fueron de 26,9 y $15,3 \%$, respectivamente. La información de clima se obtuvo de la estación meteorológica portátil WSGP1 (marca AT Delta-T Devices, Cambridge, UK) instalada en la parcela. El agua de riego utilizada tuvo un $\mathrm{pH}$ de 5,9 y CE de $2 \mathrm{dS} \mathrm{m}^{-1}$ (a $20^{\circ} \mathrm{C}$ ). La determinación de la lámina de riego para el tratamiento control, se hizo a partir de la evapotranspiración potencial (ETo) calculada mediante la ecuación de Penman-Monteith (Allen et al., 1998). El porcentaje de área sombreada medida de los árboles fue del 43\%.

El diseño experimental utilizado fue de bloques completos al azar, con cuatro repeticiones por tratamiento: la parcela elemental estuvo formada por 4 a 5 filas de 3, 4 o 5 árboles cada una.

Durante los meses de diciembre, enero y febrero, se aplicaron los siguientes tratamientos diferenciales:

Control: tratamiento regado durante todo el año al $100 \%$ de la ETc.

T1: tratamiento regado con una lámina fija correspondiente al $67 \%$ del control, durante el periodo del 28 de diciembre al 29 de febrero, coincidiendo con la fase de crecimiento rápido del fruto.

T2: tratamiento regado con una lámina fija correspondiente al 55\% del control, durante el mismo periodo del T1.

Los árboles se regaron por goteo, con seis goteros por planta de $8 \mathrm{~L} \mathrm{~h}^{-1}$ cada uno. El agua aplicada, se midió mediante contadores volumétricos. El volumen de agua aplicado a cada tratamiento se controló variando la duración del tiempo de rie- go, manteniendo igual la frecuencia, que osciló entre 2 y 3 d por semana. Las prácticas culturales llevadas a cabo fueron las comunes para el cultivo en la zona.

El potencial matricial de agua en el suelo $\Psi$ s, fue determinado con 10, 13 y 13 sensores de matriz granular (Watermark modelo 200ss; Watermark, Riverside, CA) en los tratamientos control, T1 y T2, respectivamente, instalados a $30 \mathrm{~cm}$ de profundidad y separados a $25 \mathrm{~cm}$ de la línea de goteo.

El potencial hídrico foliar se midió con una cámara de presión modelo 600 de Soil Moisture Equipment Corp. (Santa Barbara, CA), según el procedimiento descrito por Scholander et al. (1965) y siguiendo las precauciones descritas por Turner (1981). Se hicieron medidas en hojas a medio día solar, cubiertas $2 \mathrm{~h}$ antes con bolsas de plástico de cierre hermético recubiertas con papel de aluminio ( $\Psi \mathrm{t})$. Se utilizaron tres hojas por árbol, en dos árboles representativos por tratamiento, situadas en el tercio inferior en la cara norte del árbol. Las determinaciones se realizaron al comienzo de las restricciones y posteriormente cada $15 \mathrm{~d}$.

Para la medida del crecimiento del fruto, se seleccionaron 48 frutos por tratamiento, 24 en la dirección norte y 24 en la dirección sur, y se midió el diámetro ecuatorial y longitudinal, con un calibrador manual (precisión 0,01 mm) cada $15 \mathrm{~d}$, desde el 8 de enero de 2012 hasta la cosecha en abril de 2012. Las curvas de crecimiento fueron ajustadas a una ecuación logística de tres parámetros.

La tasa absoluta de crecimiento (TAC) representa un cambio de tamaño por unidad de tiempo, correspondiendo a la primera derivada del crecimiento con respecto al tiempo (Hunt, 2003). Ecuación 1.

$T A C=\frac{\delta}{\delta \mathrm{t}}$ 
Donde:

$d L / d t$ : es la derivada de la dimensión evaluada con respecto al tiempo.

La tasa de crecimiento relativo (TCR), expresa el crecimiento en términos de tasa de incremento en tamaño por unidad de tamaño (Hunt, 2003). Ecuación 2.

$T C R=\left(\frac{1}{\mathrm{~L}}\right)\left(\frac{\delta \mathrm{L}}{\delta \mathrm{t}}\right)$

Donde:

$L$ : es la dimensión evaluada.

La medición de la producción se hizo mediante la recolección comercial de la cosecha, en los árboles de cada parcela, de la cual se determinó la distribución de calibres por diámetro. Los análisis de calidad de fruta se realizaron en el laboratorio de poscosecha del Departamento de Ingeniería Civil e Ingeniería Agrícola de la Universidad Nacional de Colombia, incluyendo azúcares solubles totales ( ${ }^{\circ}$ Brix) con refractómetro digital portátil (modelo DR201-95, Krüss Optronic, Hamburgo, Alemania), acidez total titulable con $\mathrm{NaOH}$ como reactivo, índice de madurez, resistencia a la penetración con el analizador de textura de Brookfield Engi- neering Labs. (Middleboro, MA) a una velocidad de prueba de $1,5 \mathrm{mms}^{-1}$, con punta de $2 \mathrm{~mm}$. Y el color con el equipo Chromameter CR400 Konica Minolta (Tokyo), en una muestra de 20 frutos por tratamiento.

El análisis estadístico se realizó mediante el programa IBM SPSS Statistics 20 (released 2011, IBM SPSS Statistics for Windows, Version 20.0; IBM Corporation, Armonk, NY) mediante análisis de varianza (Anova) y prueba de comparación multiple DMS, con nivel de significancia del $5 \%$.

\section{RESULTADOS Y DISCUSIÓN}

\section{Volumen de agua aplicado}

Durante el periodo de restricción, la precipitación total (P) fue 53,8 mm, la (ETc), calculada según Penman-Monteith (Allen et al., 1998), fue 2,84 en enero y $3,17 \mathrm{~mm} \mathrm{dia}^{-1}$ en febrero, el agua aplicada mediante riego fue de $683(42,4 \mathrm{~mm})$, 460 (28,9 mm) y 377 L/árbol (23,6 mm), para el control, T1 y T2, respectivamente (figura 1). La reducción de riego representó un ahorro de agua en el periodo de restricción de 33 y $45 \%$ para T1 y $\mathrm{T} 2$, respectivamente.

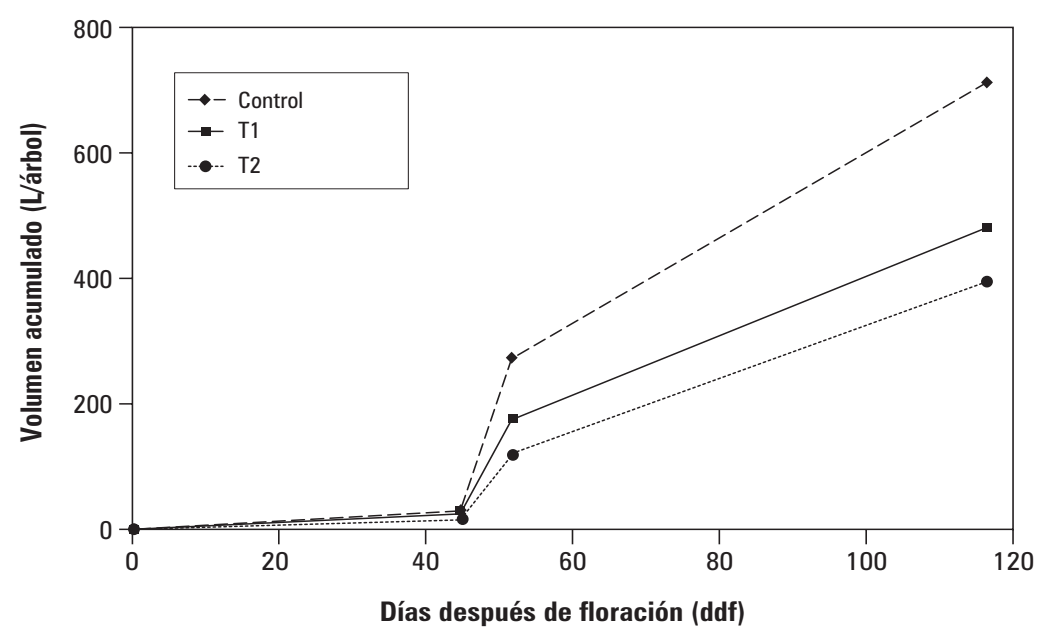

Figura 1. Volumen acumulado de agua durante el periodo de restricción para los tratamientos control (100\% de ETc), T1 (lámina de agua fija, $67 \%$ del control) y T2 (lámina de agua fija, 55\% del control). 


\section{Crecimiento del fruto}

El crecimiento del fruto durante el experimento siguió una curva sigmoide simple que describe tres fases (Fischer et al., 2012). La fase uno caracterizada por la división celular desde la floración hasta 49 días después de floración (ddf), la dos en la que se presenta alargamiento celular desde 50 hasta 110 ddf y la tres de maduración y poco crecimiento desde 111 ddf hasta la cosecha. Durante el crecimiento no se presentó diferencia significativa $(P>0,05)$ en el diámetro longitudinal y ecuatorial del fruto (figuras $2 \mathrm{~A}$ y $\mathrm{B}$ ).

En la figura 2A se observa que el crecimiento longitudinal del fruto para el T2 tiende a ser menor durante el periodo de restricción, mientras que en la etapa de recuperación alcanza el mayor crecimiento, debido a que los frutos experimentan un crecimiento compensatorio al reanudar el riego tras un déficit hídrico (Sánchez-Blanco y Torrecillas, 1995). Esto también puede indicar que los frutos son capaces de acumular materia seca durante los periodos de estrés hídrico, que puede quedar disponible para propiciar el crecimiento compensatorio, similares resultados han encontrado en pera (Wu et al., 2013) y cítricos (Cohen y Goell, 1984; Domingo et al., 1996; Vé- lez et al., 2007a). Otro factor que puede incidir para que el tamaño del fruto en un tratamiento deficitario sea mayor, es que tenga un menor número de frutos (Marsal et al., 2012), lo que no ocurrió en nuestro caso.

En el diámetro longitudinal del fruto la ecuación 4, es la que más se ajusta.

$$
y=\frac{a}{1+b * e^{-c x}}
$$

Donde: $a$, es el diámetro máximo longitudinal final del fruto, $b$, es constante de proporcionalidad y $c$, la tasa absoluta de crecimiento en los puntos de inflexión (tabla 1).

El crecimiento del diámetro ecuatorial del fruto presentó su mejor ajuste a una ecuación logística de tres parámetros, con coeficientes de determinación mayores a 0,97.

$y=\frac{a}{1+e^{-b(x-c)}}$

Donde: $y$ es dimensión del fruto; $a$, máxima dimensión final del fruto; $b$, tasa absoluta de crecimiento en el punto de inflexión y $c$, día en que se presenta la mayor tasa absoluta de crecimiento.

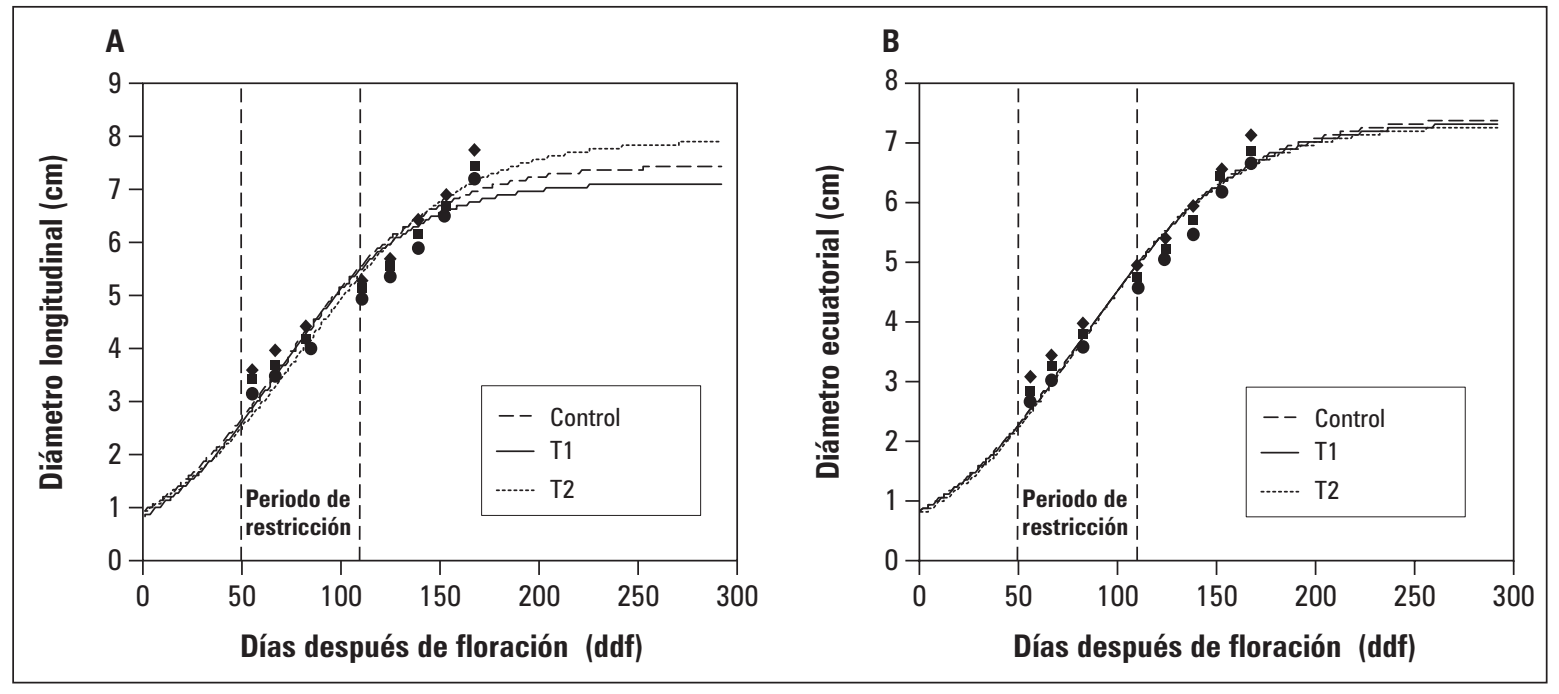

Figura 2. Curvas de crecimiento del diámetro longitudinal (A) y ecuatorial (B) del fruto de pera para los tratamientos control (100\% de ETc), T1 (lámina de agua fija, 67\% del control) y T2 (lámina de agua fija, 55\% del control). 
Tabla 1. Ecuaciones de regresión para cada modelo de crecimiento del diámetro longitudinal y ecuatorial de los frutos.

\begin{tabular}{|c|c|c|}
\hline \multicolumn{1}{|c|}{ Tratamiento } & Modelo diámetro longitudinal & Modelo diámetro ecuatorial \\
\hline Control (100\% de ETc) & $y=\frac{7,455}{1+7,044 e^{-0,0275 x}}$ & $y=\frac{7,4458}{1+e^{-0,0252(x-82,47)}}$ \\
\hline T1 (lámina de agua fija, 67\% del control) & $y=\frac{7,143}{1+7,612 e^{-0,0294 x}}$ & $y=\frac{7,3718}{1+e^{-0,0255(x-81,6962)}}$ \\
\hline T2 (lámina de agua fija, 55\% del control) & $y=\frac{7,937}{1+7,669 e^{-0,0253 x}}$ & $y=\frac{7,3104}{1+e^{-0,0262(x-81,655)}}$ \\
\hline
\end{tabular}

A partir de los modelos, la fase uno finaliza con un diámetro ecuatorial de fruto de 2,24; 2,23 y $2,18 \mathrm{~cm}$; la dos con 4,96; 4,96 y 4,95 cm y la tres con 6,$65 ; 6,61$ y $6,60 \mathrm{~cm}$, para el control, T1 y T2, respectivamente. Valores similares al control encontraron Arenas et al. (2013). Mientras que para el diámetro longitudinal la fase uno finaliza con 2,$63 ; 2,54$ y $2,46 \mathrm{~cm}$; la dos con 5,$55 ; 5,49$ y $5,38 \mathrm{~cm}$ y la tres con 6,$95 ; 6,76$ y $7,13 \mathrm{~cm}$ para el control, T1 y T2, respectivamente.

La diferencia entre modelos de crecimiento de diámetro ecuatorial y longitudinal se explica por la forma típica alargada de la pera y se puede visualizar con la relación diámetro longitudinal/ diámetro ecuatorial, que disminuye entre 50 y 125 ddf, se recupera levemente entre 125 y 139 ddf en los tratamientos $\mathrm{T} 2 \mathrm{y}$ control, finalmente se incrementa en los 153 a 167 ddf, lo que se traduce en un mayor crecimiento longitudinal, acercándose de forma gradual a la unidad y, por último, se presenta una recuperación leve del diámetro longitudinal, definiendo la forma ligeramente alargada de la era (figura 3).

\section{Tasa absoluta de crecimiento del fruto}

Durante el periodo de restricción (50-110 ddf) los modelos ajustados de tasa absoluta de crecimiento del fruto (TAC) tienen la misma

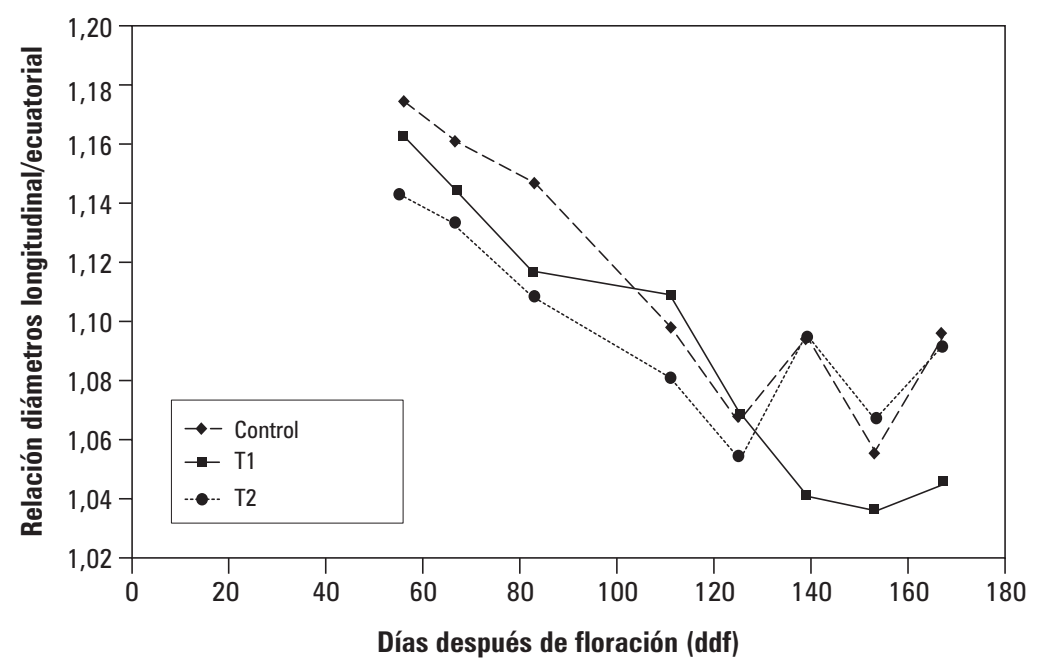

Figura 3. Relación diámetros longitudinal/ecuatorial del fruto para los tratamientos control (100\% de ETc), T1 (lámina de agua fija, $67 \%$ del control) y T2 (lámina de agua fija, $55 \%$ del control). 
tendencia de las tasas de crecimiento, además muestran el comportamiento típico para un fruto de pera, como lo encontrado en manzana por Yuri et al. (2011), en donde la máxima TAC se presentó en la fase dos, lo que coincide con lo encontrado en champa por Álvarez-Herrera et al. (2009).

En el periodo de reanudación del riego al 100\% de ETc, 15 d después de terminar el periodo de restricción se observa la tendencia de disminución de la TAC del diámetro longitudinal del fruto en el T1 y T2, mientras que en el control, se mantiene por encima (figura $4 \mathrm{~A}$, tabla 2).

En cuanto a la TAC del diámetro ecuatorial de fruto se observa un comportamiento similar a la del longitudinal, donde el máximo valor de la TAC se alcanza durante el periodo de restricción (figura 4B, tabla 3).

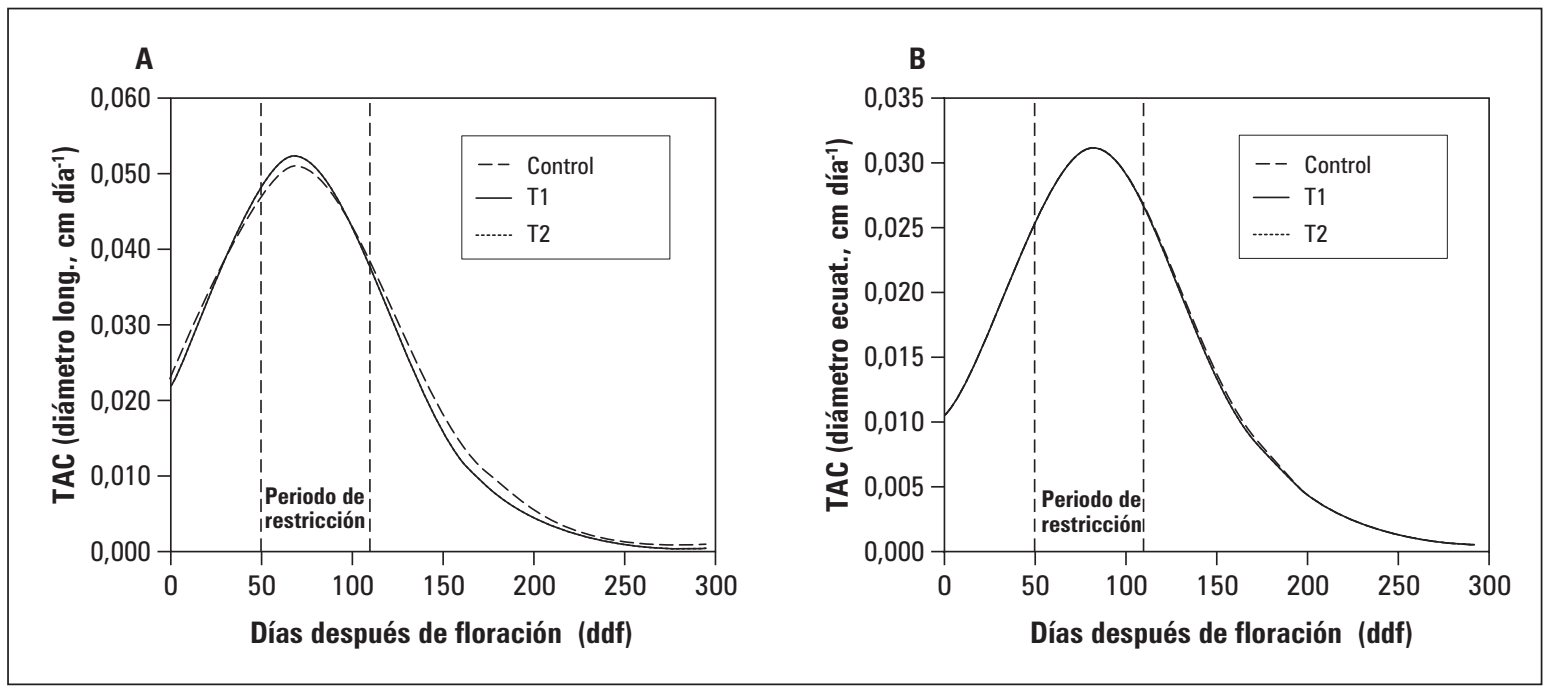

Figura 4. Tasa absoluta de crecimiento (TAC) del diámetro longitudinal (A) y ecuatorial (B) del fruto para los tratamientos control (100\% de ETc), T1 (lámina de agua fija, $67 \%$ del control) y T2 (lámina de agua fija, $\mathbf{5 5 \%}$ del control).

Tabla 2. Ecuaciones modelo de la TAC del diámetro longitudinal de los frutos.

\begin{tabular}{|l|c|}
\hline \multicolumn{1}{|c|}{ Tratamiento } & Modelo TAC diámetro longitudinal \\
\hline T0 (control, 100\% de ETc) & $T A C_{\text {DiamLT0 }}=\frac{1,467 * e^{-0,028 x}}{\left(1+7,044 * e^{-0,028 x}\right)^{2}}$ \\
\hline T1 (lámina de agua fija, 67\% del control) & $T A C_{\text {DiamLT1 }}=\frac{1,576 * e^{-0,029 x}}{\left(1+7,612 * e^{-0,029 x}\right)^{2}}$ \\
\hline T2 (lámina de agua fija, 55\% del control) & $T A C_{\text {DiamLT2 }}=\frac{1,521 * e^{-0,025 x}}{\left(1+7,669 * e^{-0,025 x}\right)^{2}}$ \\
\hline
\end{tabular}


Tabla 3. Ecuaciones del modelo de la TAC del diámetro ecuatorial de los frutos.

\begin{tabular}{|c|c|c|}
\hline Tratamiento & & odelo TAC diámetro ecuatorial \\
\hline \multirow{2}{*}{ T0 (control, 100\% de ETc) } & \multirow{2}{*}{$T A C_{\text {DiamET0 }}=$} & $\left(0,186 * e^{0,025(x+82,47)}\right)$ \\
\hline & & $2 * e^{0,025(x+82,47)}+e^{0,05 x}+e^{4,12}$ \\
\hline \multirow{2}{*}{ T1 (lámina de agua fija, 67\% del control) } & \multirow{2}{*}{$T A C_{\text {DiamET1 }}=$} & $\left(0,192 * e^{0,025(x+81,696)}\right)$ \\
\hline & & $2 * e^{0,025(x+82,47)}+e^{0,052 x}+e^{4,248}$ \\
\hline \multirow{2}{*}{ T2 (lámina de agua fija, 55\% del control) } & \multirow{2}{*}{$T A C_{\text {DiamET2 }}=$} & $\left(0,190 * e^{0,026(x+81,655)}\right)$ \\
\hline & & $2 * e^{0,026(x+81,655)}+e^{0,052 x}+e^{4,246}$ \\
\hline
\end{tabular}

\section{Tasa relativa de crecimiento del fruto}

La tasa relativa de crecimiento (TRC) tanto de diámetro ecuatorial como longitudinal se inicia con una disminución lenta en la fase uno de crecimiento, seguido por una disminución drástica en la fase dos hasta la cosecha, similar a lo encontrado en champa por Álvarez-Herrera et al. (2009), y diferente a lo encontrado en manzano por Yuri et al. (2011), en el cual la disminución es constante durante todas las fases de crecimiento (figuras $5 \mathrm{~A}$ y $\mathrm{B}$, tablas 4 y 5 ).

\section{Relaciones hídricas}

El crecimiento de frutos presentó un comportamiento acorde a las aplicaciones de las láminas de agua para cada uno de los tratamientos. El Is del tratamiento control se mantuvo durante la restricción entre -20 y $-60 \mathrm{kPa}$, mayor al T1 y
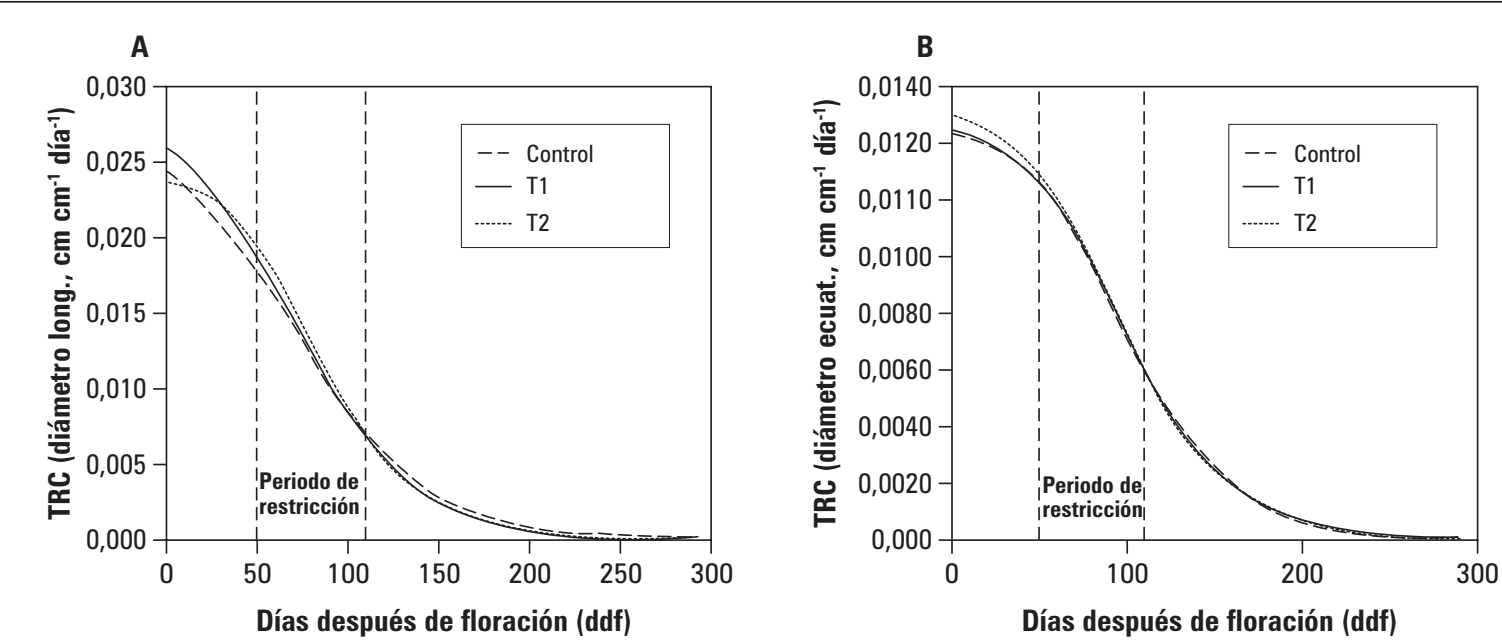

Figura 5. Tasa de crecimiento relativo (TCR) del diámetro longitudinal (A) y ecuatorial (B) de fruto para los tratamientos control (100\% de ETc), T1 (lámina de agua fija, $67 \%$ del control) y T2 (lámina de agua fija, $55 \%$ del control). 
Tabla 4. Ecuaciones del modelo de la tasa de crecimiento relativo (TCR) del diámetro longitudinal de fruto para los tratamientos control (100\% de ETc), T1 (lámina de agua fija, $67 \%$ del control) y T2 (lámina de agua fija, $55 \%$ del control).

\begin{tabular}{|c|}
\hline Modelo TCR diámetro longitudinal \\
\hline$T R C_{\text {Diam TT }}=\frac{1+7,044 e^{-0,0275 x}}{7,455} * \frac{1,467 * e^{0,028 x}}{\left(1+7,044 * \mathrm{e}^{-0,028 x}\right)^{2}}$ \\
\hline$T R C_{\text {DiamLT1 }}=\frac{1+7,612 e^{-0,0294 x}}{7,143} * \frac{1,576 * e^{0,029 x}}{\left(1+7,612 * \mathrm{e}^{-0,029 x}\right)^{2}}$ \\
$T C_{\text {DiamLT2 }}=\frac{1+7,669 e^{-0,025 x}}{7,937} * \frac{1,521 * e^{0,025 x}}{\left(1+7,669 * \mathrm{e}^{-0,025 x}\right)^{2}}$
\end{tabular}

Tabla 5. Ecuaciones del modelo de la tasa de crecimiento relativo (TCR) del diámetro ecuatorial de fruto para los tratamientos control (100\% de ETc), T1 (lámina de agua fija, $67 \%$ del control) y T2 (lámina de agua fija, $55 \%$ del control).

\begin{tabular}{|c|c|c|}
\hline \multicolumn{3}{|c|}{ Modelo TCR diámetro ecuatorial } \\
\hline \multirow{2}{*}{$T R C_{\text {DiamET0 }}=$} & $1+e^{-0,025(x-82,47)}$ & $0,186 * e^{0,025(x+82,47)}$ \\
\hline & 7,4458 & $2 * e^{0,025(x+82,47)}+e^{2 * 0,025 x}+e^{2 * 0,025 * 82,47}$ \\
\hline \multirow{2}{*}{$T R C_{\text {DiamET1 }}=$} & $1+e^{-0,025(x-81,6962)}$ & $0,192 * e^{0,026(x+81,696)}$ \\
\hline & 7,372 & $2 * e^{0,026(x+81,696)}+e^{2 * 0,026 x}+e^{2 * 0,026 * 81,696}$ \\
\hline \multirow{2}{*}{$T R C_{\text {DiamET2 }}=$} & $1+e^{-0,026(x-81,655)}$ & $0,190 * e^{0,026(x+81,655)}$ \\
\hline & 7,310 & $2 * e^{0,026(x+81,655)}+e^{2 * 0,026 x}+e^{2 * 0,026 * 81,655}$ \\
\hline
\end{tabular}

T2, siendo más negativo a mayor restricción y se igualaron cuando se presentaron las lluvias. El tratamiento T2 alcanzó el menor potencial (-101 $\mathrm{KPa})$ en el 92 DDF con una recuperación lenta al final de los tratamientos (MolinaOchoa et al., 2015).

Con la imposición del déficit hídrico los $\Psi \mathrm{t}$, tal como era esperable, tendieron a disminuir. E1 T2, al que se le aplicó la menor lámina, tuvo menores valores que el control y $\mathrm{T} 1$ sin diferencias significativas $(P>0,05)$, alcanzando un $\Psi$ t de $-0,65$ $\mathrm{MPa}$, después de $33 \mathrm{~d}$ de iniciarse el recorte de agua (83DDF) (Molina-Ochoa et al., 2015), que se ajusta a lo que define De Swaef et al. (2009) para la mayoría de los frutales (entre - 0,5 y 1,5 $\mathrm{MPa}$ ) y muy superior a los encontrados por Marsal et al. (2012), (-1,2 MPa, en pera var. Conferencia) y Naor et al. (2006) (-2,2 MPa, en pera var. Spadona), sin afectar el rendimiento.

No hubo diferencias significativas $(P>0,05)$ en la producción, número y peso de frutos por árbol y los parámetros de calidad, debido posiblemente a que la reducción del volumen de agua en los tratamientos deficitarios no fue severa (Molina- 
Ochoa et al., 2015). Sin embargo el tratamiento control tuvo una producción igual al T2 y mayor $4,35 \%$ que el T1, pero no significativa.

El tratamiento control resultó con el mayor peso medio de fruto (147 g). E1 T2, con el mayor número de frutos por árbol $(32,5)$, tuvo el menor peso promedio por fruto (141 g), lo que confirma el efecto de la competencia entre el peso del fruto y el número de frutos por árbol, independiente del estrés de agua (Kriedemann y Barrs 1981; Agusti et al., 2003).

La producción obtenida en los tratamientos de riego fue baja, comparada con lo encontrado por Arenas (2011) en la misma parcela, esto puede indicar que 'Triunfo de Viena' presenta alternancia en su producción.

\section{CONCLUSIONES}

Las tres láminas de riego aplicadas durante la etapa de crecimiento rápido del fruto, no mostraron diferencias significativas en producción y calidad de la fruta, lo que quiere decir que se puede hacer un uso más eficiente del agua.

El crecimiento del diámetro longitudinal y ecuatorial del fruto tuvo un comportamiento sigmoide simple, obteniendo a partir de los datos experimentales un modelo logístico, mediante el cual se logra la determinación de la tasa absoluta y relativa de crecimiento, que es importante al momento de predecir el tiempo de la cosecha, que en nuestro caso fue de 167 ddf.

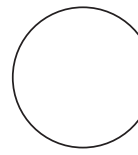

Agustí, M., A. Martínez-Fuentes, C. Mesejo, J. Mariano y V. Almela. 2003. Cuajado y desarrollo de los frutos cítricos. Série Divulgació Técnica. Generalitat Valenciana, Conselleria D'Agricultura, Peixca y Alimentació, Valencia, España.

Alegre, S., J. Marsal, M. Mata, A. Arbonés y J. Girona. 2002. Regulated deficit irrigation in olive tres (Olea europea L., cv. Arbequina) for oil production. Acta Hortic. 586, 259-262.

Allen, R.G., L.S. Pereira, D. Raes y M. Smith. 1998. Crop evapotranspiration, guidelines for computing crop water requirements. Irrigation and Drainage Paper 56. FAO, Roma.

Álvarez-Herrera, J.G., J.A. Galvis y H.E. Balaguera-López. 2009. Determinación de cambios físicos y químicos durante la maduración de frutos de champa (Campomanesia lineatifolia R. \& P.). Agron. Colomb. 27(2), 253-259.

Arenas-Bautista, M.C., J.E. Vélez y J.H. Camacho. 2013. Crecimiento del fruto de peral bajo el sistema de
REFERENCIAS BIBLIOGRÁFICAS

riego por goteo con una y dos líneas. Rev. Colomb. Cienc. Hortic. 6(2), 140-151. Doi: 10.17584/ rcch.2012v6i2.1972

Arenas-Bautista, M.C. 2011. Efecto de la utilización de doble línea de riego por goteo en el cultivo de pera (Pyrus communis L.) variedad Triunfo de Viena. Tesis de maestría. Facultad de Ingeniería, Universidad Nacional de Colombia, Bogotá.

Behboudian M.H., G.S. Lawes y K.M. Griffiths. 1994. The influence of water deficit on water relations, photosynthesis and fruit growth in Asian pear (Pyrus serotina Rehd.). Sci. Hortic. 60, 89-99. Doi: 10.1016/0304-4238(94)90064-7

Casierra, F., D.I. Hernández, P. Lüdders y G. Ebert. 2003. Crecimiento de frutos y ramas de manzano 'Anna' (Malus domestica Borkh.) cultivado en los altiplanos colombianos. Agron. Colomb. 21(1-2), 69-73.

Casierra-Posada, F. 2012. Manzano y peral (Malus domestica Borkh. y Pyrus communis L.). pp. 657-681. En: Fischer, G. (ed.) Manual para el cultivo de frutales en el trópico. Produmedios, Bogotá. 
Chalmers, D. J., G. Burge, P.H. Jerie y P.D. Mitchell.1986. The mechanism of regulation of 'Barlett' pear fruit and vegetative growth by irrigation withholding and regulated deficit irrigation. J. Amer. Soc. Hort. Sci.111(6), 904-907

Cohen, A. y A. Goell. 1984. Fruit development as an indicator of the irrigation needs of citrus trees. Proc. Int. Soc. Citric. Congr. (Sao Paulo) 1, 114-121

De Swaef, T., K. Steppe y R. Lemeur. 2009. Determining reference values for stem wáter potential and máximum daily trunk shrinkage in young apple trees based on plant responses to water deficit. Agr. Water Manage. 96, 541-550. Doi: 10.1016/j. agwat.2008.09.013

Domingo, R., M. Ruiz-Sánchez, M. Sánchez-Blanco y A. Torrecillas. 1996. Water relations, growth and yield of Fino lemon trees under regulated deficit irrigation. Irrig. Sci. 16, 115-123. Doi: 10.1007/ BF02215619

Ebel, R.C., E.L. Proebsting y R.G. Evans. 1995. Deficit irrigation to control vegetative growth in apple and monitoring fruit growth to schedule irrigation. HortScience 30, 1229-1232.

Fischer, G., F. Ramírez y P.J. Almanza-Merchán. 2012. Inducción floral, floración y desarrollo del fruto. pp. 120-140. En: Fischer, G. (ed.). Manual para el cultivo de frutales. Produmedios, Bogotá.

Girona, J. 1996. Estrategia de Riego deficitario controlado para la mejora de la eficiencia en el uso de aplicación del agua de riego. Frut. Prof. 80, 32-38.

Goldschmidt E.E. y S.P. Monselise. 1977. Physiological assumptions toward the development of a citrus fruiting model. Proc. Int. Soc. Citriculture 2, 668672.

Huguet, J.G. 1985. Appréciation del' état hydrique d'une plante á partir des variations micrométriques de la dimension des fruits ou des tiges au cours de la journée. Agronomie 5, 733-741.

Hunt, R. 1990. Basic growth analysis. Plant growth analysis for beginners. Unwin Hyman, Boston, MA.

Hunt, R. 2003. Growth analysis, individual plants. Encyclopedia of plant sciences. Academic Press, London.

Intrigliolo, D.S. y J.R. Castel. 2006. Performance of various water stress indicators for prediction of fruit size response to deficit irrigation in plum. Agric. Water Manage. 173-180. Doi: 10.1016/j. agwat.2005.12.005
Jackson, J.E., 2003. Biology of horticultural crops. The biology of apples and pears. Cambridge university press. New York, NY.

Klepper, B., D.V. Browning y H.M. Taylor. 1971. Stem diameter in relation to plant water status. Plant Physiol. 48, 683-685. Doi: 10.1104/pp.48.6.683

Kozlowski, T.T. 1967. Diurnal variations in stem diameter of small trees. Bot. Gaz. 123, 60-68.

Kozlowski, T.T. y C.H. Winguet. 1964. Diurnal and seasonal variation in radio of tree stems. Ecology 45, 149-155

Kriedemann, P.E. y H.D. Barrs. 1981. Citrus orchards. pp. 325-417. En: Kozlowsky, T.T. (ed.). Water deficits and plant growth. Vol. 6. Academic Press, New York, NY.

Levy, Y., J. Shalhevet y H. Bielorai. 1979. Effect of irrigation regime and water salinity on grapefruit quality. J. Amer. Soc. Hort. Sci. 104, 356-359

Li, X., T. Wang, B. Zhou, W. Gao, J. Cao y L. Huang. 2014. Chemical composition and antioxidant and anti-inflammatory potential of peels and flesh from 10 different pear varieties (Pyrus spp.). Food Chem. 152, 531-538. Doi: 10.1016/j.foodchem.2013.12.010

Marsal, J. G. Lopez, J. Del Campo, M. Mata, A. Arbones y J. Girona. 2010. Postharvest regulated deficit irrigation in 'Summit' sweet cherry: fruit yield and quality in the following season. Irrig. Sci. 28(2), 181-189. Doi: 10.1007/s00271-009-0174-z

Marsal, J., M. Mata, A. Arbones, J. Rufat y J. Girona. 2002. Regulated deficit irrigation and rectification of irrigation scheduling in young pear trees: an evaluation based on vegetative and productive response. Eur. J. Agron. 17, 111-122. Doi: 10.1016/ S1161-0301(02)00002-3

Marsal, J., G. López, M. Mata y J. Girona. 2012. Postharvest deficit irrigation in 'Conference' pear: Effects on subsequent yield and fruit quality. Agric. Water Manage. 103, 1-7. Doi: 10.1016/j.agwat.2011.10.012

Molina-Ochoa, M.J., J.E. Vélez-Sánchez y A. GalindoEgea. 2015. Resultados preliminares del efecto del riego deficitario durante el periodo de crecimiento rápido del fruto de pera (var. Triunfo de Viena) en la producción y calidad del fruto. Rev. Colomb. Cienc. Hortic. 9(1), 38-45. Doi: 10.17584/ rcch.2015v9i1.3744

Naor, A., R. Stern, M.A. Flaishman, Y. Gal y M. Peres. 2006. Effects of postharvest water stress on autumnal bloom and subsequent-season produc- 
tivity in mid-season 'Spadona' pear. J. Hort. Sci. Biotechnol. 81, 365-370.

Ningbo C., D. Taisheng, L. Fusheng, T. Ling, W. Mixia, L. Xiaozhi., L. Zhijun y K. Shaozhong. 2009. Response of vegetative growth and fruit development to regulated deficit irrigation at different growth stages of pear-jujube tree. Agric. Water Manage. 96, 1237-1246. Doi: 10.1016/j. agwat.2009.03.015

Sánchez-Blanco, M.J. y A. Torrecillas. 1995. Aspectos relacionados con la utilización de estrategias de riego deficitario controlado en cultivos leñosos. Riego deficitario controlado. Fundamentos y aplicaciones. Ed. Mundi-Prensa, Madrid.

Scholander, P.F., H.T. Hammel. E.D. Bradstreet y E.A. Hemmingsen. 1965. Sap pressure in vascular plants. Sci. 184, 339-346.

Shalhevet J, A. Mantell, H. Bielorai y D. Shimshi. 1979. Irrigation of field and orchard crops under semiarid conditions. International Irrigation Information Center, Volcani Center, Bet Dagan, Israel.

Turner, N.C. 1981. Techniques and experimental approaches for the measurement of plant water status. Plant Soil. 58, 339-336. Doi: 10.1007/ BF02180062

Vélez, J., D.S. Intrigliolo y J.R. Castel. 2007a. Scheduling deficit irrigation of citrus trees with maximum daily trunk shrinkage. Agric. Water Manage. 90(3), 197-20.

Vélez, J., D.S. Intrigliolo y J.R. Castel. 2007b. Programación del riego deficitario en Clementina de Nules, mediante Dendrómetros. International Magazine of Citrus 38, 313-317.

Vélez-Sánchez, J.E., D.S. Intrigliolo y J.R. Castel. 2011. Programación de riego en base a sensores de medida del estado hídrico del suelo y de la planta. Rev. UDCA Actual. Divulg. Cient. 14(2), 197-20.

Wu, Y., Z. Zhao, W. Wang, Y. Ma y X. Huang. 2013. Yield and growth of mature pear trees under water deficit during slow fruit growth stages in sparse planting orchard. Sci. Hortic. 164, 189-195. Doi: 10.1016/j.scienta.2013.09.025

Yuri, J.A., J. González, J. Verdugo y A. Del Pozo. 2011. Responses of fruit growth, quality, and productivity to crop load in apple cv. Ultra Red Gala/ MM111. Sci. Hortic. 127, 305-312. Doi: 10.1016/j. scienta.2010.10.021 